\title{
LF-ViT: Development of a Virtual Reality Guided Tour Mobile App of Landmark University Teaching and Research Farm
}

\author{
https://doi.org/10.3991/ijim.v13i05.9119 \\ Emmanuel Oluwatobi Asani ${ }^{(凶)}$, Mmesoma Zuri Chidioke, \\ Ayoola John Shoyombo, Aderonke Anthonia Kayode \\ Landmark University, Omu-Aran, Nigeria \\ asani.emmanuel@lmu.edu.ng \\ Azubuike Ezenwoke \\ Covenant University, Ota, Nigeria \\ Foluke Okocha \\ Landmark University, Omu-Aran, Nigeria
}

\begin{abstract}
In this work, we designed and developed a Virtual Reality guided tour mobile app for Landmark University farms, LF-ViT. We were motivated by the need to circumvent the problem of bio-security caused by incessant visit to the farm by visitors, tourists or customers.

The guided tour was implemented using the storytelling technique. Other technical details of the design and implementation process are discussed.
\end{abstract}

Keywords - Virtual Reality, guided tour, storytelling technique, mobile app

\section{$1 \quad$ Introduction}

The possibility of representing and replacing real places, objects and activities with an immersive virtual experience holds great potentials for wide ranging application areas and is consequently generating increased research interest. Virtual Reality (VR) is very important for obvious reasons. One such reason, is the seeming impossibility of recreating some real-life scenario or events. It may be impossible for instance, to recreate the reaction of an expert pilot in crisis situation to a pilot training. This can be recreated using virtual reality. Historical events, which of course cannot be reversed, can also be experienced vividly through Virtual Reality. Places, sites or sceneries which are otherwise inaccessible, owing to distance or other constraining factors have made the need for the creation of a virtual environment using computer generated imagery data of the real or supposed environment necessary. Places or events which are otherwise non-existent in the real world may also be imagined, simulated and experienced virtually. 
Virtual Reality, otherwise referred to as 'immersive multimedia' or computersimulated reality, deploys technology to replicates an environment which may be real or hypothetical, and integrates an interactive simulation if the user's physical presence and environment. Virtual Reality artificially induces the sensory experience of the user. According to Loomis et al., (1999), immersive virtual experiences offers the possibility to combine the benefits of both real-life and virtual environments. Horan (1997) depicts the VR experience based on user's perspective pictorially as comprising of Environment tools, virtual environment, devices and other environments. The environment tools are models and resources for the virtual reality experience (See Figure 1).

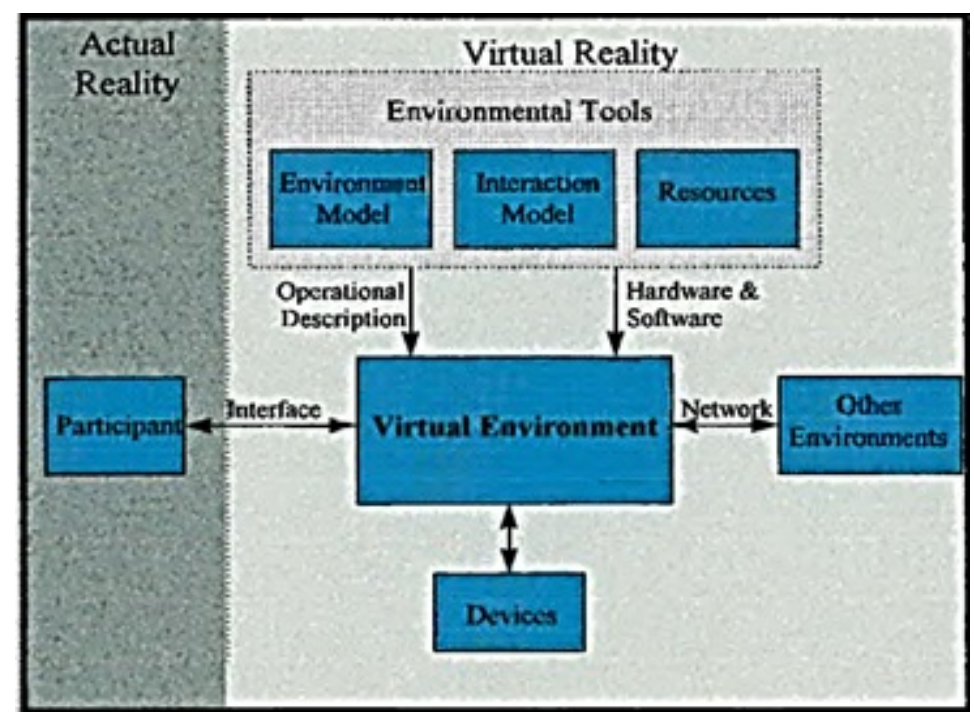

Fig. 1. The Virtual Reality Model (Horan,1997)

Applications of Virtual Reality or Augmented Reality which spans fields such as simulation, education, entertainment, medical and game evolved from efforts that dates as far back as 1800. Heilig's sensoroma in 1956 however marked a milestone in the development of virtual reality. Advances in Virtual Reality researches have resulted in considerable improvement with the development of interactive computer technology and sophisticated 3D modelling packages (Noh et al, 2009). Such advances are revolutionizing tourism and advertisement as it is now possible to provide immersive experience of concepts or places being propagated. This shift in advertising paradigm tends to eliminate distraction and disruption of explorative experience arising from frequent visual reference to mobile devices (Phil et al, 2018).

In this study, we modelled and developed a virtual environment of landmark university teaching and research farm namely Landmark Farms Virtual Tour (LF-ViT). Incessant visits to the farm, especially by tourists or customers who in most cases are non-practitioners can potentially compromise the bio-security of the farm; we were motivated by the need to circumvent this situation by replacing actual visits with vir- 
tual experience. The virtual system has an integrated virtual tour guide that would guide tourist by describing the physical features of the farm. This system would enhance the knowledge of tourist who are not visually impaired on how the farm looks like in reality without physically being there.

The remainder of this paper is organized as follows: Section 2 reviews related works on Virtual Reality Technology that are relevant to our method. Section 3 presents an overview of our work detailing the design and modelling technique used. Section 4 provides details on the implementation strategy and tools deployed for our system while Section 5 concludes the paper.

\section{Review of Related Works}

VR technology has been deployed in wide ranging application areas such as in education and training, architecture, medicine, manufacturing, entertainment etc. (Zaihui Cao and Zhongyan hu, 2012). While VR has been used in tourism and indeed holds a great potential for the industry, little has been reported about this in literature.

Most VR in tourism employ either interactive experience, illustration or story telling method. For instance, the VR system developed by Wojciechowski et al. (2004) depicts the interactive experience method with the use of 3D models. Users, usually content modelers can use authoring tools to model exhibitions virtually by arranging objects efficiently based on in-built templates. C-MAP, a location-aware tour guide app by Sumi et al (1998), on the other hand, provides personalized assistance to exhibition tourists by providing useful animated illustrative information. C-MAP captures users' spatial-temporal information and interests ("mental context") to provide them dynamic contextual guide. The app also features integrated offline and online information sharing services to tourists.

Galean (1995) proposed the "river analogy" model of virtual environment traversal in place of the traditional "branching analogy". The traditional "branching analogy" model involves the sequential interlacing of presentation in the virtual system. He opined that virtual guided tour systems whose narration and presentation structure rely on user's interaction with its virtual environment is based on the "emergent narration" model. The proposed method models a river-like flow in the virtual route traversal. The user is like a boat traversing the virtual landscape. Unlike the branching model, the flow is seamless as it traverses the multi-levelled structure.

Pollefeys et al, (2001) developed a virtual reality system that helps users take a guided tour of reconstructed Sagalassos. Sagalassos was a Mediterranean city that existed roughly from the 4th to 7 th century. This became necessary because tourists visiting the archaeological reconstruction site stands a risk of compromising the originality of the site. The process may also be cumbersome thus leading to boredom and likelihood of missing important monuments. The system allows interaction between the user and the virtual environment. Users can also get on-demand guidance by asking the virtual tour using automatic speech recognition. Similarly, Younes et al., (2017), developed a fully immersive system to recreate the Roman Theater at Byblos. Byblos, existed around (around 8000-4000 BCE) (Dunand, 1954). It is a great ar- 
chaeological site generating interests among tourist. However due to its ruin, tourist may miss its monumental features. Thus, the need to recreate it virtually.

(Papaioannou et al., 2003), proposed model to enhance the virtual experience of users in viewing archaeological sites. To achieve this, efforts were made to optimize the performance of existing techniques that have been used successfully in gaming and advertisement. Some are of improvements considered include visual quality as it affects illumination (sequential or dynamic), memory use, video overlay and image processing. Speed was also improved. Use cases were also considered to demonstrate the improvements achieved. Most of the styles are intuitive. However, we invite you to read carefully the brief description below.

\section{LF-ViT: Project Overview}

Our VR tour system (LF-ViT) has three major components as proposed by (Tanriverdi and Jacob, 2001) which are "application, interface and dialog control". The whole of LF-ViT is the application component. Everything about LF-ViT ranging from logic, knowledge base, features and modules is considered. The interface component is the view from where users are able to access and manipulate the system. Users receive data and communicate with the system through the interface. The dialog control is the communication module that makes interaction between users and the interface objects possible.

LF-ViT is a Virtual Reality guided tour mobile app for Landmark University teaching and research farm. The flow of interlacing components is based on the river analogy as proposed by Galean (1995) and the narrative is emergent by design. In developing this system, we set three objectives which include:

- To design a virtual environment of Landmark University Farm

- To develop a virtual environment of Landmark University Farm

- To integrate a virtual tour guide functionality

In achieving our objectives, we did a physical mapping of the farm in which panoramic and sky view photos were taken, google map was also used to ensure that structures are placed accurately in the virtual environment. Blender was used to model the objects and various components into $3 \mathrm{D}$. C\# programming language was used to program the object behavior in the game engine unity $3 \mathrm{D}$.

\subsection{Design}

Because the application is meant to portray reality, it is necessary to avoid unrealistic functions like constraining the user movement to a predefined path. The system was designed to be a free wandering tour experience but this also affected the storytelling functionality. Each building in the farm had defined audio narratives so that when a user enters the building a recorded voice of an actor would be played to describe features of the building and animals in it. In designing the system, we em- 
ployed the two phased VRID model as proposed by Tanriverdi and Jacob (2001). They are high-level design phase and the low-level design phase.

High Level Design: There three major activities in the High-level design phase:

- HLA1. Identification of Data entities

- HLA2. Identification of Objects

- HLA3. Constructing the objects models

- HLA3.1. Graphics

- HLA3.2. Object Properties

- HLA3.3. Interacting components

- HLA3.4. Internal communications (mediator)

- HLA3.5. External communications

HLA1: Discovering the data elements: Data elements enable interaction between VR system interface and external entities of the VR system. The inflows of data into the VR can be from either of the following sources:

- Users

- Peripherals

- Other VR systems

In this case, the only data element is the 3D coordinate of the mobile device communicated to the interface by the gyroscope.

HLA2: Identification of objects: The process of identifying object involves the following:

- Elicitation of candidate objects from the interface description

- Identification and choosing valid objects

- Classification of objects as either virtual or physical

Virtual objects are those objects that are to modeled and generated by the computer while physical objects are the physical components that interact both internally and externally with the VR system. In this case the physical object is the Mobile device. The virtual objects to be modelled are buildings, birds, trees, fences, fowls, cattle, tractors and other legal objects identified in the farm.

HLA3: Modelling the objects: The goal in this activity is to model the virtual objects identified in HLA2. In achieving this, we consider the specification/features below:

i. Graphical models: This involves a high-level description of graphics needs of virtual objects. They are:

- Graphical model of the exterior of buildings needed

- Graphical model of the interior of buildings needed

- Minimum amount of texturing details of the building needed

- Graphical model of the farm animals needed with minimum vertices count.

- Graphical model of the trees should be placed at different locations in the farm

- Graphical model of birds to fly around the farm should be created 
- Each building in the farm must have colliders to prevent the player from passing through the wall.

ii. Object property: The behaviors exhibited by some objects are summarized in the table below:

Table 1. Behavior of Virtual Objects

\begin{tabular}{|l|l|}
\hline \multicolumn{1}{|c|}{ Objects } & \multicolumn{1}{c|}{ Behaviors } \\
\hline Poultry & Pecking, standing, walking \\
\hline Birds & Flying, standing, jumping, perching, screaming \\
\hline Pig & Grunting, walking, standing \\
\hline Cow & Walking, standing, mooing \\
\hline Goat & Bleating, walking, standing \\
\hline
\end{tabular}

iii. Interactions: Users interact with the virtual environment using the gaze input module.

iv. External communications: In this system, communication inflows into the virtual farm are $3 \mathrm{D}$ coordinates coming from the mobile device. There are no communications outflows.

Low-level (LL) design phase: The Low-level design phase takes as input, the output of the high-level design. The modelling activities are carried out at a lower level of abstraction to generate more design specifications:

LL1: Graphics: Low-level graphics design maps graphical models to objects properties. We show the 3D model of the farm in Fig 2, geotagging in Fig 3 and UV mapping and texturing in Fig 4.

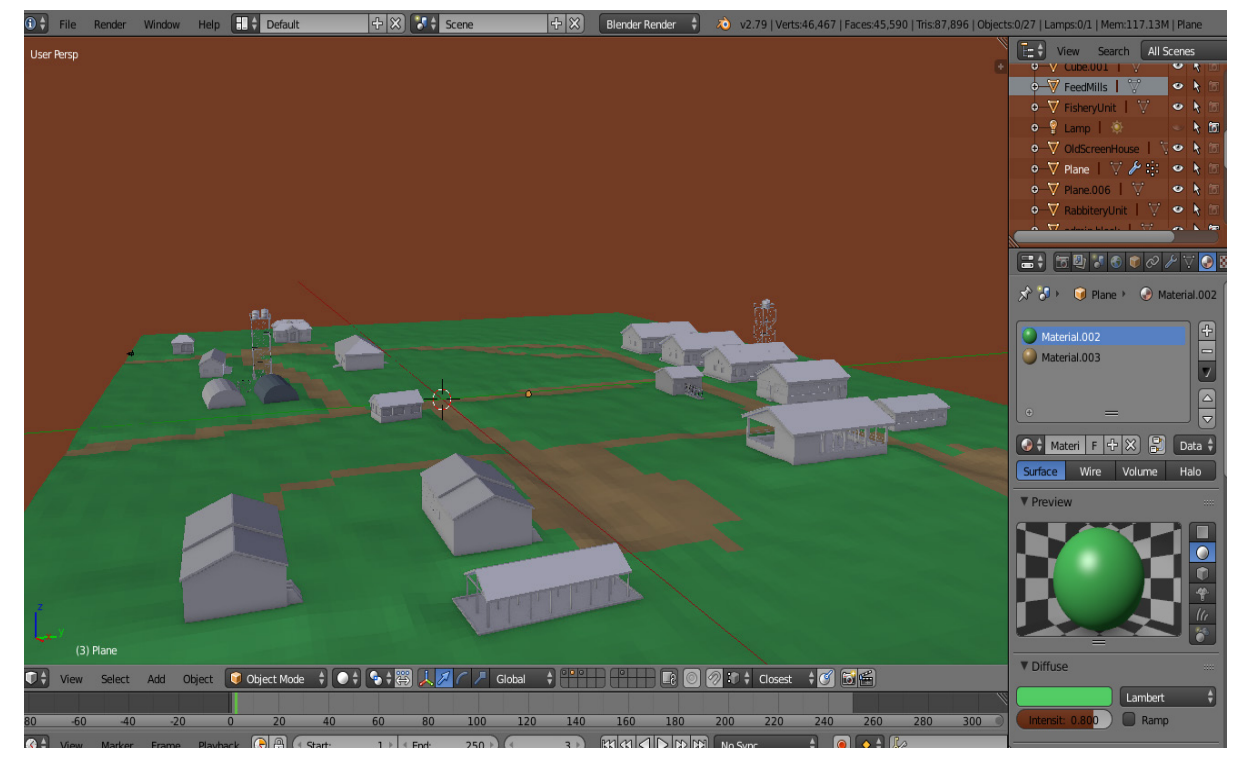

Fig. 2. 3D Model of the Farm 


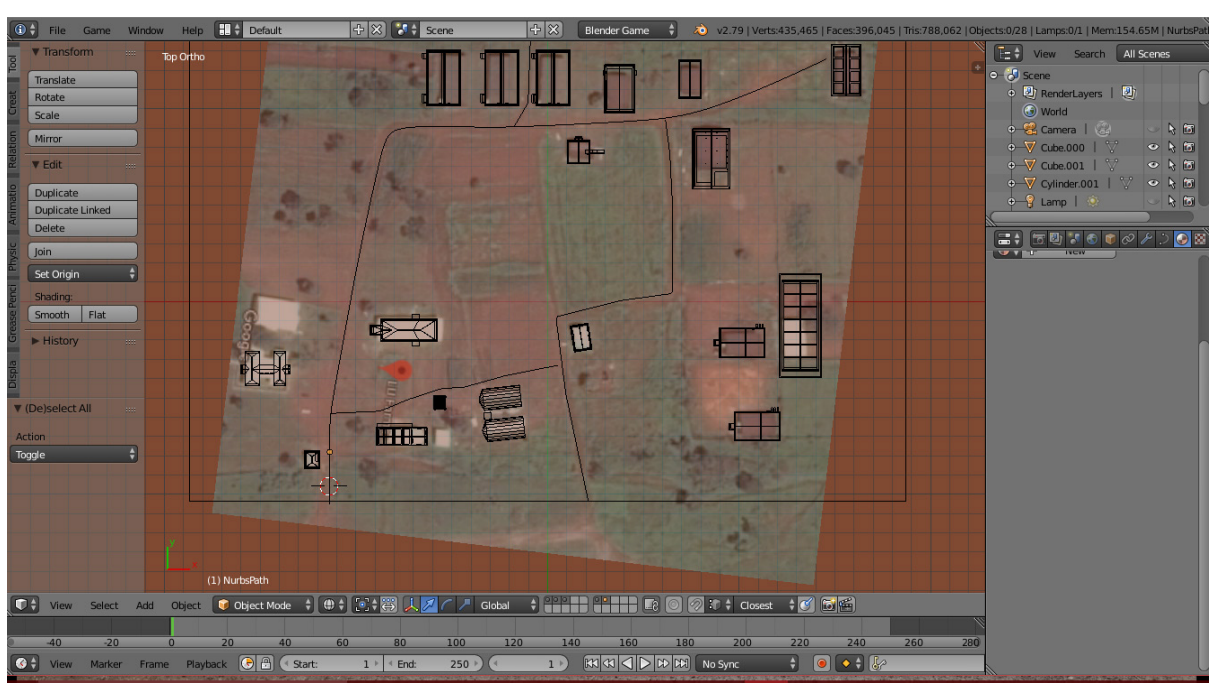

Fig. 3. Placement of Farm Objects in their correct geo-location with the aid of Google Map

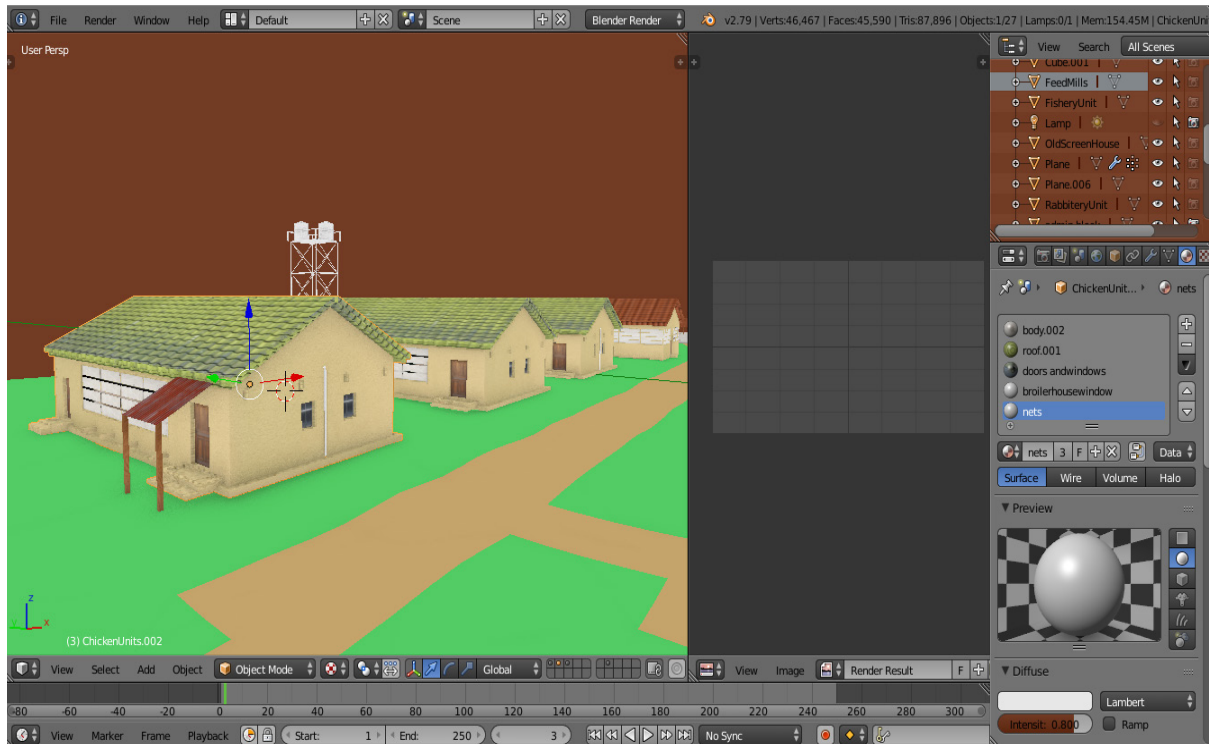

Fig. 4. Fig 4. Definition of UV Mapping and Texturing

LL2: Behaviors: In low-level design of behaviors, the goal is to formalize finegrained procedural details of behaviors that have been specified in HL (see Figure 5). 


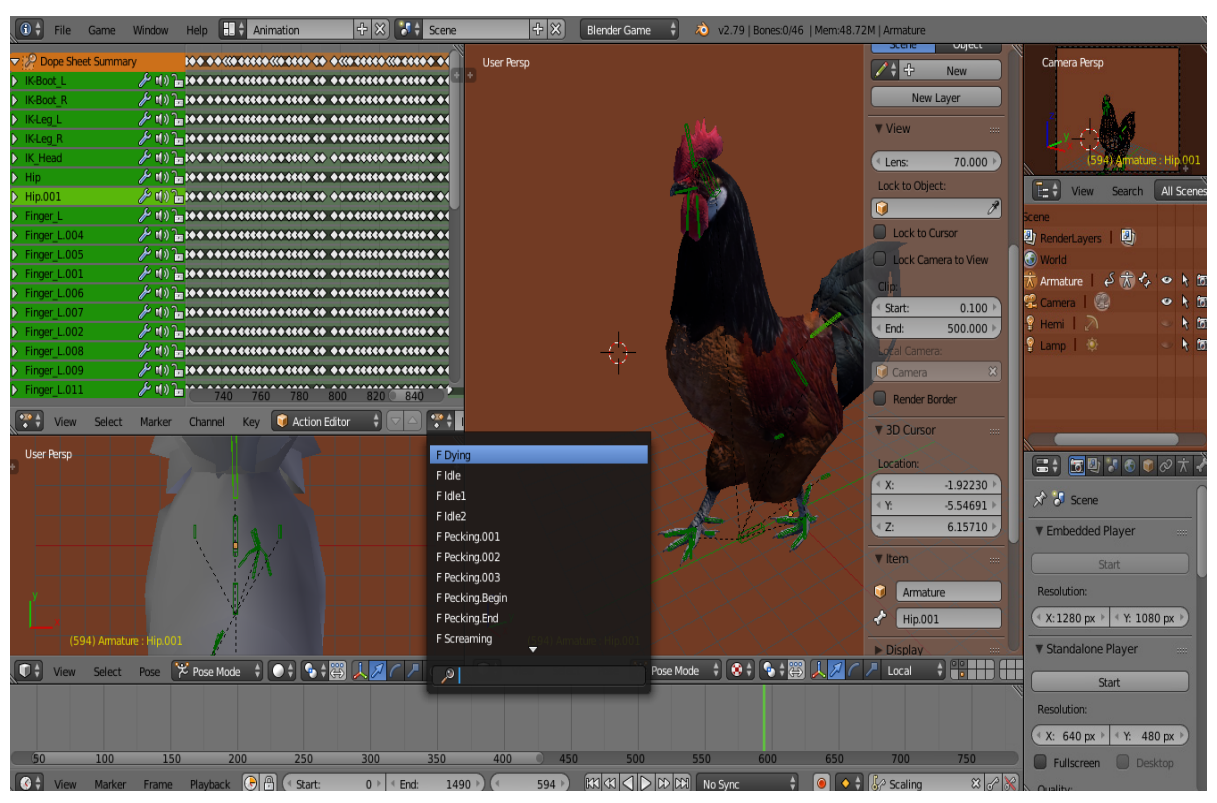

Fig. 5. Associated Behavior being defined in 3D Model

LL3: Interactions: Timed Gaze input module: The gaze input is the communication module of the system. It is therefore the dialog control. The gaze input algorithm implemented in C\#, snippets are represented below:

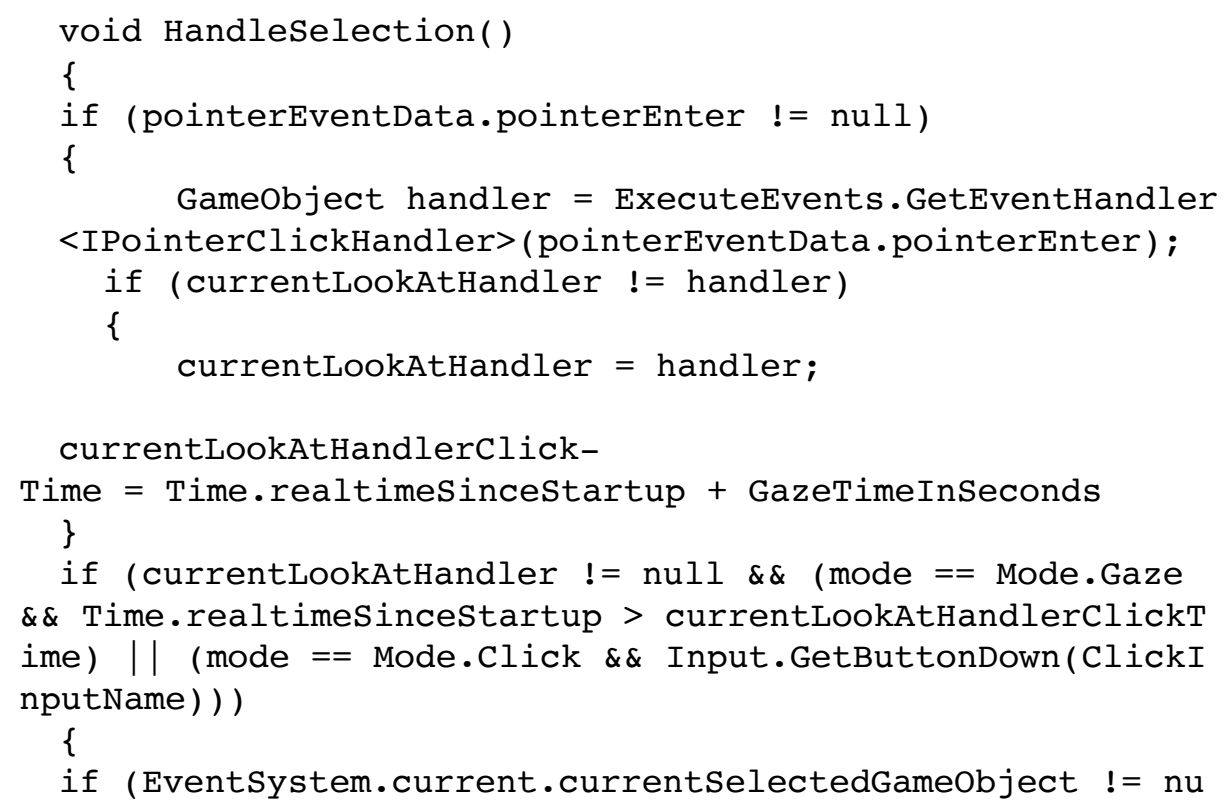




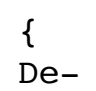

LL4: Internal communications (mediator): The objective here is to put in place a scheduling routine to managing the communication requests. This is achieved by unity's in-built event system and event trigger. Event triggers can be applied to game object with colliders which allow for raycasting.

LL5: External communications: The objective here is to put in place a routine for coordinating the message passing system among the communicating objects. In this case communication between the game objects and the gyroscope is achieved with the gaze input module.

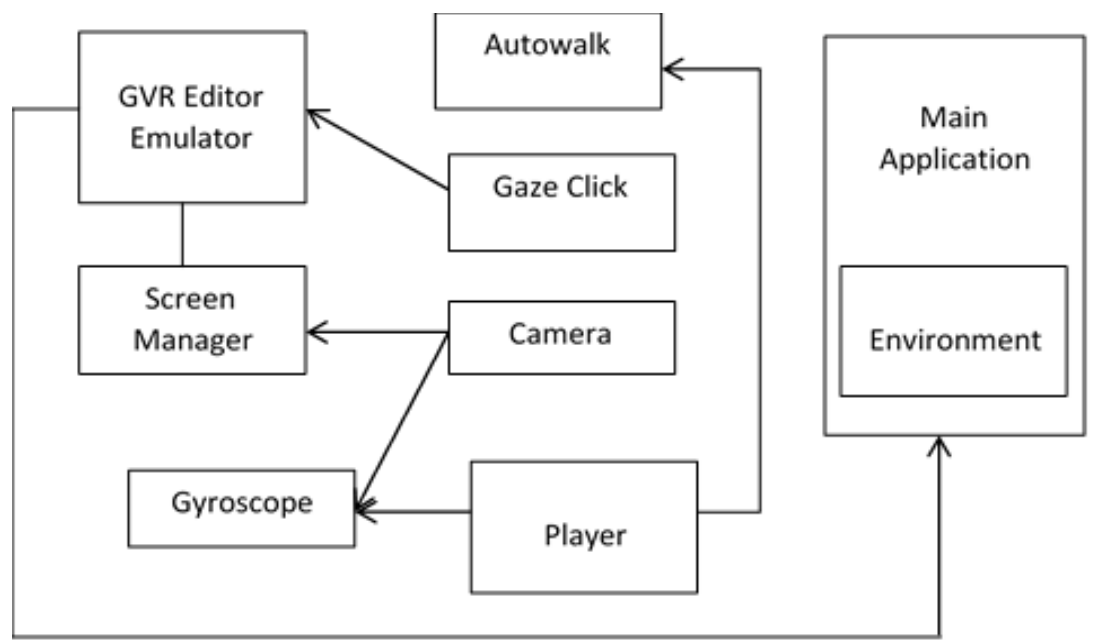


Fig. 6. Class Diagram showing interaction among the objects of the system

The class diagram in Figure 6 shows the interaction among the objects of the system. The player connects the application object which is the gyroscope via the gaze click module. The gaze click is connected to the GVR editor emulator which controls the screen manager. Autowalk increments the player's location in the cartesian plane. The application provides the virtual environment.

\section{Implementation}

The Implementation involves the creation of a version of the application which involves the integration of all the assets developed in the design Phase into one working system. This phase was directed by some content experts in fields such as: Agriculture, Audio Engineering, and 3D modelling.

The app interface describes the user interface of the virtual reality system.

Figure 7 describes the first scene of the virtual reality app where the player is to choose whether to begin the tour or not using gaze input.

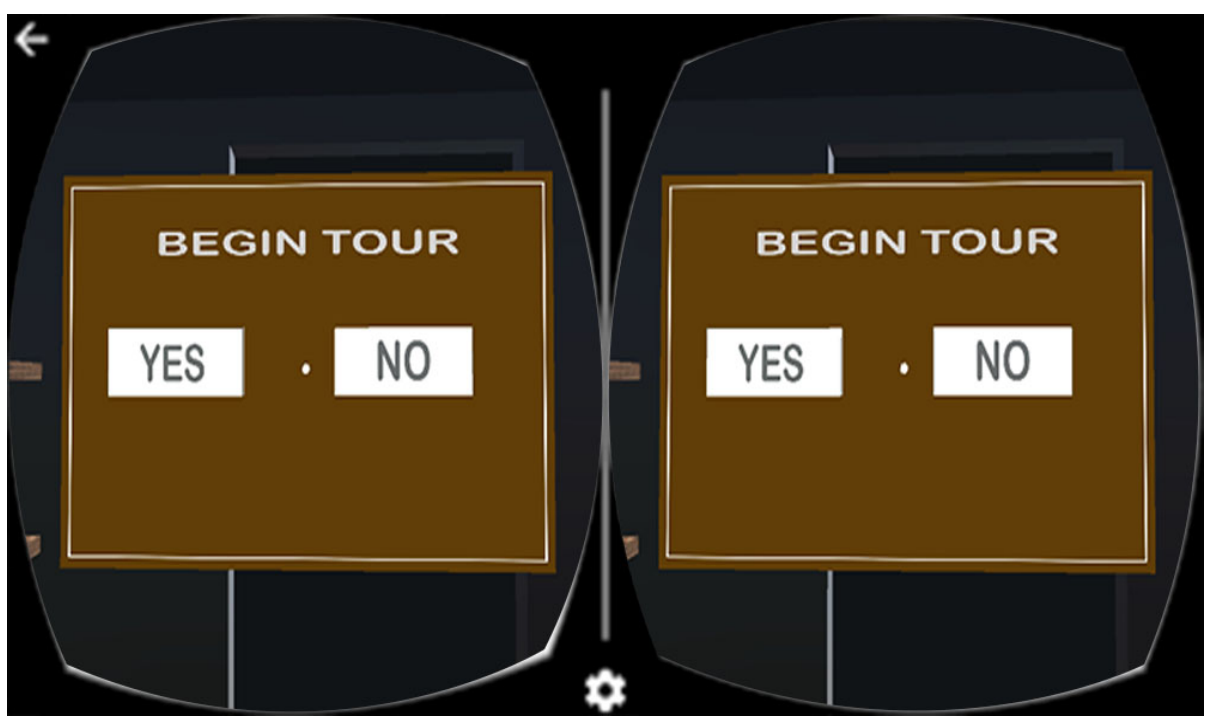

Fig. 7. Main Menu User Interface

The gaze input is timed to immerse the user into the virtual farm environment after few seconds of gazing at 'ÝES' in the first scene as shown in Figure 8. 


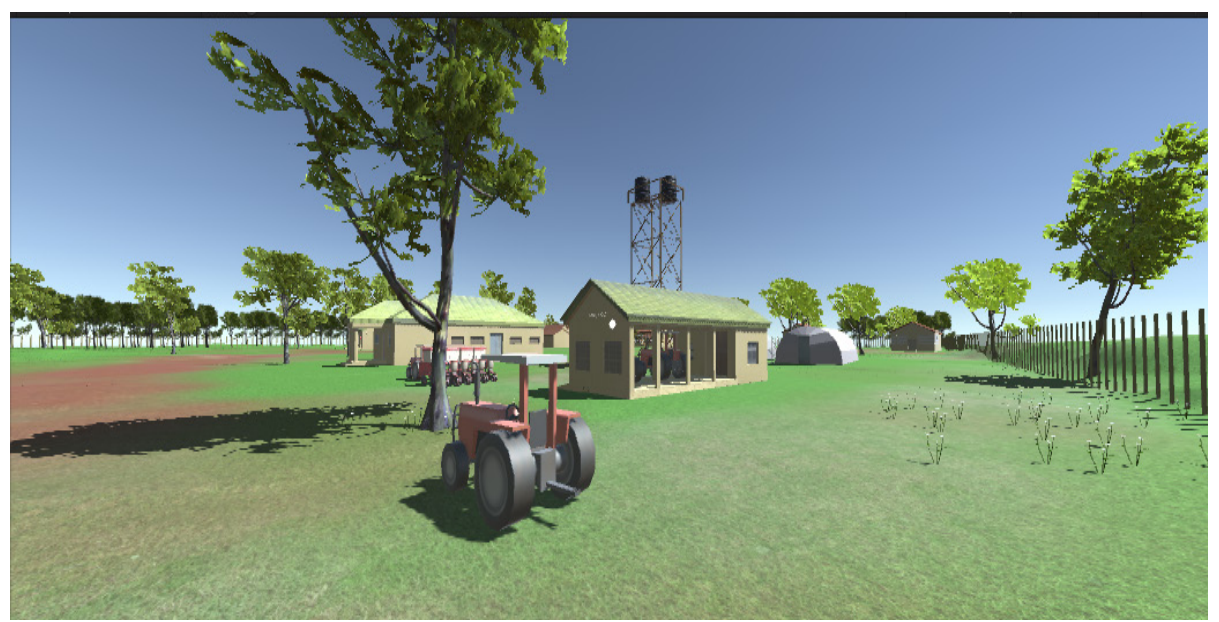

Fig. 8. Virtual Farm Environment

'Flying birds' character have been modelled into the virtual environment as shown in Figure 9. The birds tend to follow the player around the farm by maintaining some given distances once the main farm level of the VR app is loaded.

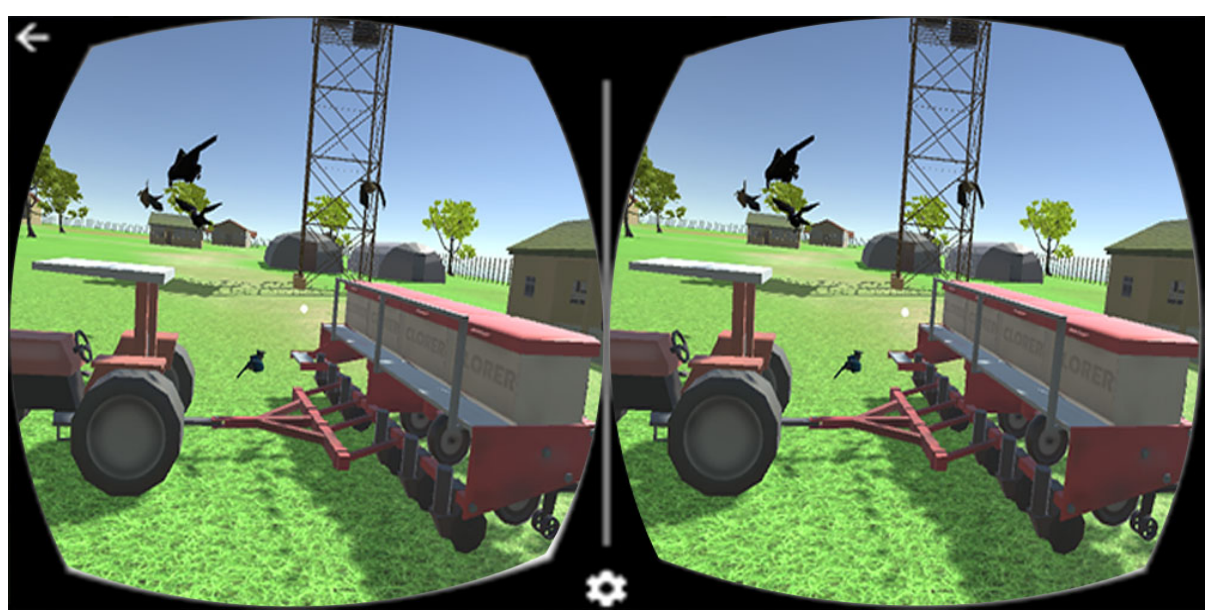

Fig. 9. Virtual Environment showing flying birds' character

Figure 10 describes the method for entering buildings. The enter building UI is located on the door of the buildings. Once the UI is gazed upon by the player the inside building level is generated where a voice over describes features of that building and the animated animals in it. 


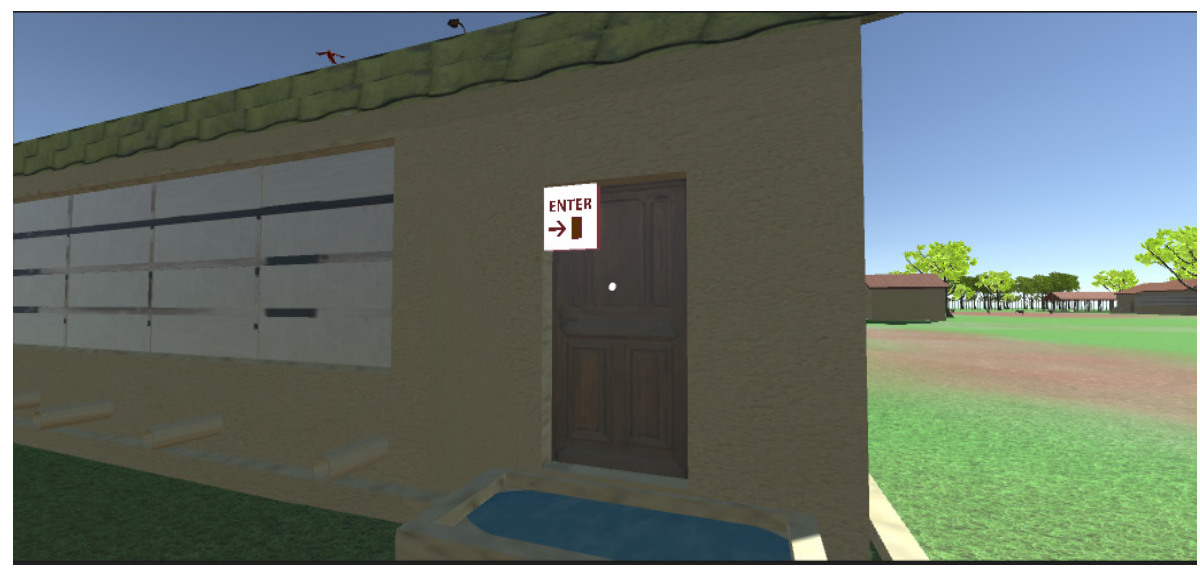

Fig. 10.User Interface for Entering building

Figure 11 describes the method for exiting the building. When the player gazes at the button the main menu level is loaded.

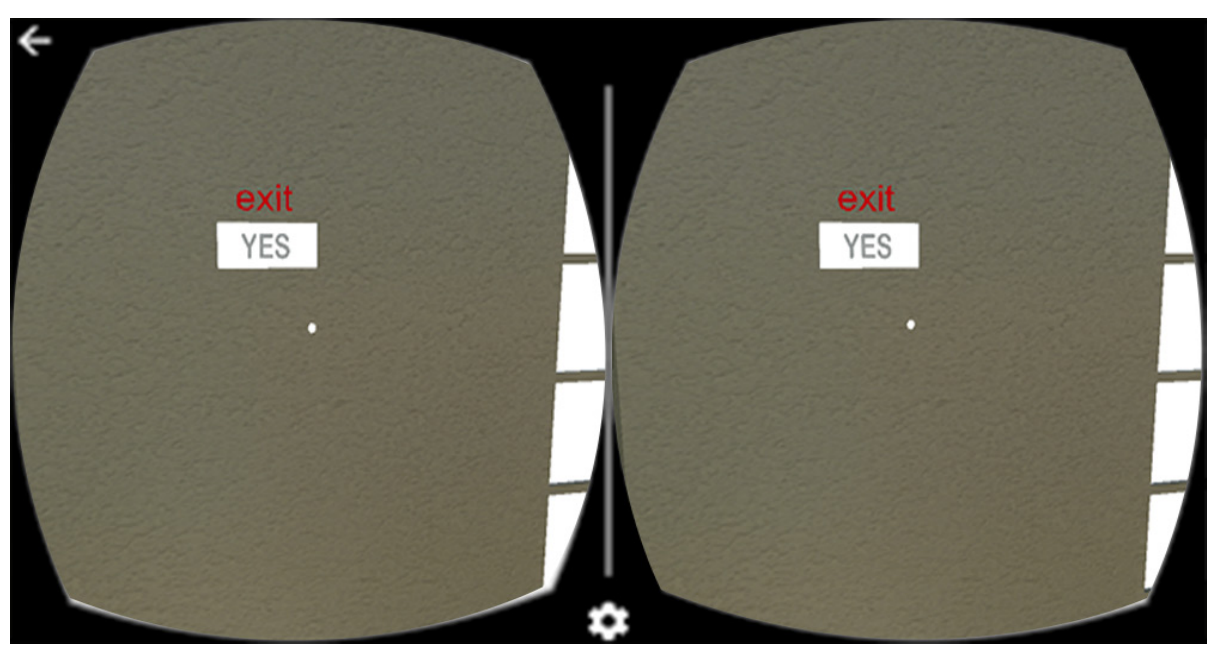

Fig. 11.User Interface for exiting building

\section{Conclusion}

LF-ViT demonstrated a river analogy based virtual tour guide of a Landmark University Teaching and Research Farm running on a mobile device which can be viewed via a head-mounted device. It is also a demonstration of the alternate usefulness of Virtual Reality and its potentials for commercial purpose. The system relied on voice over and gaze input to help users explore the farm. With the development of this system, farm owners, especially those that deal in fresh produce and livestock can show- 
case their products vividly to potential buyers without them compromising the biosecurity of the farm with the customers' physical presence.

NOTE: The app documentation and code files can be downloaded on github.com.

The project link is: https://github.com/asanitobiloba/LF-ViT

The .apk can be downloaded on dropbox: https://www.dropbox.com/s/2 muu1lfk39rqne6/LF-ViT.apk?dl=0

\section{References}

[1] Galyean Tinsley A. (1995). Guided navigation of virtual environments. In Proceeding: The 1995 symposium on Interactive 3D graphics. Pages 103-ff. ACM New York. ISBN:089791-736-7. https://doi.org/10.1145/199404.199421.

[2] Horan P (1997). "The World Is What You Make It" - An Application of Virtual Reality to the Tourism Industry. Masters' Degree thesis. Dublin City University, Dublin. Available online http://doras.dcu.ie/19501/1/Patrick P Horan 20130725161913.pdf. Accessed $\underline{14 / 06 / 18}$

[3] Loomis, J.M., Blascovich, J.J., Beall, A.C., (1999). Immersive virtual environment technology as a basic research tool in psychology Behavior Research Methods, Instruments, \& Computers (1999) 31: 557-564. https://doi.org/10.3758/BF03200735

[4] Noh, Z., Sunar, M. S., Pan, Z. (2009). A review on augmented reality for virtual heritage system. In International Conference on Technologies for E-Learning and Digital Entertainment (pp. 50-61). Springer, Berlin, Heidelberg.

[5] Papaioannou, Georgios \& Gaitatzes, Athanasios \& Christopoulos, Dimitrios. (2003). Enhancing virtual reality walkthroughs of archaeological sites. Proceedings of the 4th International conference on Virtual Reality, Archaeology and Intelligent Cultural Heritage, November 05-07, 2003, Brighton, United Kingdom. pp 175-184. doi:10.2312/VAST/VAST03/175-184. https://doi.org/10.1145/584993.585011

[6] Phil Bartie, William Mackaness, Oliver Lemon, Tiphaine Dalmas, Srini Janarthanam, Robin L. Hill, Anna Dickinson, Xingkun Liu, (2018). A dialogue based mobile virtual assistant for tourists: The SpaceBook Project. Computers, Environment and Urban Systems, Volume 67. Pp 110-123. https://doi.org/10.1016/j.compenvurbsys.2017.09.010

[7] Pollefeys, M., Van Gool L., Akkermans I., De Becker D. and Demuynck K. (2001). A guided tour to virtual Sagalassos. In Proceedings of the 2001 conference on Virtual reality, archeology, and cultural heritage (pp. 213-218). Glyfada, Greece. ACM. ISBN:1-58113447-9. https://doi.org/10.1145/584993.585027.

[8] Sumi Y., Etani T., Fels S., Simonet N., Kobayashi K., Mase K. (1998) C-MAP: Building a Context-Aware Mobile Assistant for Exhibition Tours. In: Ishida T. (eds) Community Computing and Support Systems. Lecture Notes in Computer Science, vol 1519. Springer, Berlin, Heidelberg. https://doi.org/10.1007/3-540-49247-x_10

[9] Tanriverdi, Vildan \& J. K. Jacob, Robert. (2001). VRID: A Design Model and Methodology for Developing Virtual Reality Interfaces. ACM Symposium on Virtual Reality Software and Technology, Proceedings, (VRST 2001), Banff, Canada, November 2001. Doi:10.1145/505008.505042. https://doi.org/10.1145/505040.505042

[10] Wojciechowski, R., Walczak, K., White, M., Cellary, W., (2004). Building virtual and augmented reality museum exhibitions. In Proceedings of the Ninth International Conference on 3D Web Technology. ACM, New York, NY, USA, pp. 135-144. https://doi.org/10.1145/985040.985060. 
[11] Younesa G., Kahilb R., Jalladc M., Asmara D., Elhajja I., Turkiyyahb G and Al-Harithyc H. (2017). Virtual and augmented reality for rich interaction with cultural heritage sites: A case study from the Roman Theater at Byblos. Digital Applications in Archaeology and Cultural Heritage 5(2017). pp1-9. http://dx.doi.org/10.1016/j.daach.2017.03.002

[12] Zaihui Cao and Zhongyan hu (2012). Design Virtual Reality Scene Roam for Tour Animations Base on VRML and Java. Physics Procedia 25. pp 693 - 699. ISBN 1875-3892 https://doi.org/10.1016/j.phpro.2012.03.145.

\section{$7 \quad$ Authors}

Emmanuel Oluwatobi Asani is a faculty member of the Department of Computer Science, Landmark University, Omuaran, Nigeria. His research interest bothers on modelling of mathematical and statistical theories using soft computing tools to solve cyber and information security problems. He has attended and presented papers in both local and international journal with his research efforts appearing in local and international peer reviewed journals. In August 2015, he was honoured at the iSTEAM MINTT conference held at the University of Benin for his contributions to scientific research.

Mmesoma Zuri Chidioke is a Computer Scientist with special interest in Computer Graphics and 3D animation. chijioke.mmesoma@lmu.edu.ng

Ayoola John Shoyombo is a Lecturer at the College of Agricultural Sciences, Landmark University. He is a researcher with focus on Animal Genetics and Breeding. He can be reached at shoyombo.ayoola@lmu.edu.ng. Skype: shoyombo.ayoola

Aderonke Anthonia Kayode is a faculty member of the Department of Computer Science, Landmark University, Omuaran, Nigeria. Her research interest is on Health Informatics. kayode.aderonke@lmu.edu.ng

Azubuike Ezenwoke, is an Assistant Professor in the Department of Computer and Information Sciences, Covenant University. His research interests include Artificial intelligence and intelligent systems, Data mining, and spiritual computing. azu.ezenwoke@covenantuniversity.edu.ng

Foluke Okocha is an Information professional with research interests in information system security, technology adoption and ubiquitous learning. She holds a master's degree in Information Science and has publications in learned journals. dada.foluke@lmu.edu.ng

Article submitted 2018-07-27. Resubmitted 2019-01-24. Final acceptance 2019-01-29. Final version published as submitted by the authors. 